\title{
nature
}

physics

\section{Life among peers}

The constitution of the 'physics community' was perhaps more straightforward a hundred years ago than it is now. Those working in the field published in a handful of journals and society proceedings, creating a written record of what was known, or thought to be known, at the time. Typically, journal editors - 'peers' by some description - selected the work to be published, a practice adopted by the Royal Societies of Edinburgh and London in the mid-eighteenth century ${ }^{1}$. Famously, the peer review of Albert Einstein's 1905 papers in the Annalen der Physik was in essence an endorsement by one of the journal's editors, Max Planck and Wilhelm Wien - both peers without a doubt.

However, later in the twentieth century, the sheer amount of work and

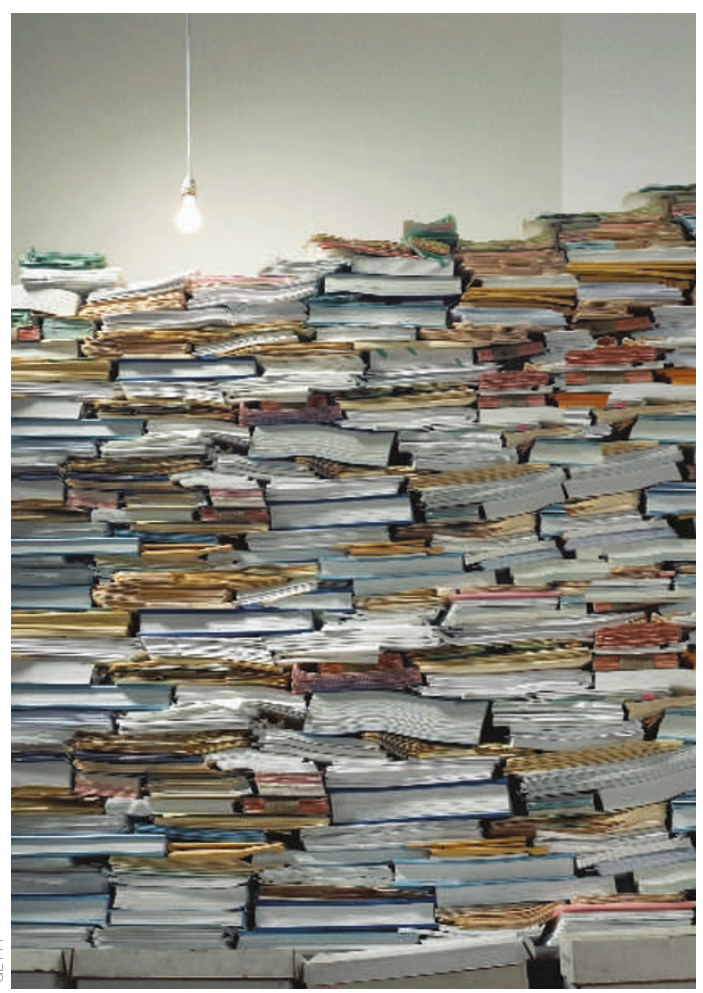
number of workers, in ever more disciplines and subdisciplines, were such that the system was bound to change. The variety of journals offering paper pages for publication expanded considerably; and in the refereeing process, the responsibilities were increasingly transferred to external experts. A necessary choice, perhaps: when John Maddox assumed the editorship of Nature in 1966, he found a backlog of more than 2,000 papers waiting for him, arranged in "fourteen monthly piles"; the need for a formal refereeing system was clear, and one was introduced soon after. As the body of knowledge grew rapidly, so did the number of 'opinion formers', shaping the published record of their community through expert peer review.

New perspectives on the reporting of results and ideas have emerged in the electronic age. The preprint server, arXiv.org, has proved a useful outlet, going far beyond what journals can do (or intend to do), and making evident the impracticability of capturing all knowledge 'the old way'. But even in the electronic world, certain limits apply. A fine balance has to be found between granting the widest possible freedom and ensuring the necessary degree of quality. Since January 2004, arXiv.org has supported a system of endorsement ${ }^{3}$ to verify that arXiv contributors belong to the scientific community - not dissimilar to the system of previous centuries, but the responsibility, and power, rests with a much broader basis of 'endorsers'.

Still, there is a plethora of information available - too much for any individual to handle. Of course, the traditional-style journals are still here Nature Physics among them - with content reflecting and communicating the choices of experts in the field through traditional peer review. It is an approach that facilitates browsing - any reader may stumble across something interesting that they would not normally read. But now the web also affords the tools for structuring, and digesting, the information at large: it's like a journal club, but with a global dimension. Sites such as Naboj ${ }^{4}$ provide a platform for open comment on all papers uploaded onto arXiv.org, allowing readers to base their choice of material on the opinions of a wide base of 'commenters'. Meanwhile, Nature's open-peer-review trial ${ }^{5}$ is exploring the same concept as part of the traditional peer review process.

Both Naboj and the Nature trial have got off to a slow start. It seems that scientists are rather shy with strangers, but probably all that's needed is more time for the idea to take hold. For instance, on

REFERENCES

1. Spier, R. Trends Biotech. 20, 357-358 (2002).

2. Maddox, J. Nature 378, 521-523 (1995)

3. http://arxiv.org/help/endorsement/

4. http://www.naboj.com/

5. http://blogs.nature.com/nature/ peerreview/trial/

6. http://philica.com/

7. http://www.plosone.org/ sites such as eBay and Amazon, customers - surely physicists among them - are more outgoing when it comes to commenting and recommending. In fact, the concept of open comment has already been formally taken on in the publishing world. The online journal Philica ${ }^{6}$ immediately accepts all articles for publication, but they are then subject to permanent anonymous review by visitors to the journal's website. PLoS ONE ${ }^{7}$, the latest offspring of the open-access publisher Public Library of Science, attempts to capture readers' insights and views in an online forum on papers it publishes after expert peer review.

Once again the notion of the 'physics community' is evolving. We have a tremendous, ever-growing treasure of knowledge, and new tools with which to render it accessible, manageable and open to discussion. Don't be shy. 